\title{
Associação de metemoglobinemia e deficiência de glicose-6-fosfato desidrogenase em pacientes com malária tratados com primaquina
}

\author{
Association of methemoglobinemia and glucose-6-phosphate \\ dehydrogenase deficiency in malaria patients \\ treated with primaquine
}

\author{
Marli Stela Santana ${ }^{1,2}$, Marcos Antonio Ferreira da Rocha ${ }^{1}$, Ana Ruth Lima Arcanjo ${ }^{1,2}$, \\ José Felipe Jardim Sardinha, ${ }^{1,2}$, Wilson Duarte Alecrim ${ }^{1}$ \\ e Maria das Graças Costa Alecrim 1,2,3
}

\begin{abstract}
RESUMO
Este estudo teve como objetivo verificar a ocorrência de metemoglobinemia em indivíduos deficientes da glicose-6-fosfato desidrogenase durante o tratamento da infecção malárica com primaquina. Foram selecionados pacientes com diagnóstico para malária por Plasmodium vivax ou mista $V+F$ (Plasmodium vivax + Plasmodium falciparum), Grupo 1: com 74 indivíduos com diagnóstico clínico de metemoglobinemia e Grupo 2: 161 indivíduos sem diagnóstico clínico de metemoglobinemia. Quanto à deficiência da G6PD, nos Grupos 1 e 2, houveram 51,3\% (38) e 8,7\% (14) de indivíduos enzimopênicos, respectivamente, demonstrando através de tais dados, significância estatística na associação com a metemoglobinemia somente nos indivíduos do Grupo 1 ( $(\mathrm{p}<0,05)$. A comparação da relação da metemoglobinemia à deficiência da G6PD mostrou haver uma possivel associação de indivíduos enzimopênicos desenvolverem metemoglobinemia com maior freqüência.
\end{abstract}

Palavras-chaves: Metemoglobinemia. Deficiência da glicose-6-fosfato desidrogenase. Malária.

\begin{abstract}
This study had the aim of investigating occurrences of methemoglobinemia among individuals with glucose-6-phosphate dehydrogenase deficiency during treatment for malaria infection using primaquine. Patients with a diagnosis of malaria caused by Plasmodium vivax or the $V+F$ mixture (Plasmodium vivax + Plasmodium falciparum) were selected. Group 1 consisted of 74 individuals with a clinical diagnosis of methemoglobinemia and Group 2 consisted of 161 individuals without a clinical diagnosis of methemoglobinemia. The glucose-6-phosphate dehydrogenase deficiency rates (numbers of enzymopenic individuals) in Groups 1 and 2 were $51.3 \%$ (38) and $8.7 \%$ (14) respectively. These data demonstrated a statistically significant association with methemoglobinemia only among the individuals in Group $1(\mathrm{p}<0.05)$. Investigation of the relationship between methemoglobinemia and glucose-6-phosphate dehydrogenase deficiency showed that there was a possible association such that enzymopenic individuals may develop methemoglobinemia more frequently.
\end{abstract}

Key-words: Methemoglobinemia. Glucose-6-phosphate dehydrogenase deficiency. Malaria.

Cerca de 350 a 500 milhões de novos casos de malária ocorrem anualmente nas áreas tropicais e subtropicais do globo, sendo a maioria destas infecções causadas pelo Plasmodium vivax e Plasmodium falciparum ${ }^{33}$. No Brasil, aproximadamente 99.5\% dos casos de malária ocorrem na região Amazônica, cujas áreas de maior transmissão são aquelas onde há condições precárias de habitação e trabalho, e encontram-se próximas a florestas e a coleções de água ${ }^{23}$.
A primaquina é a única droga antimalárica efetiva contra as formas exoeritrocitárias das quatro espécies de Plasmosdium capazes de infectar o homem, assim como efetuar cura radical, eliminando as formas latentes teciduais da infecção pelo Plasmodium vivax e Plasmodium ovale ${ }^{14}$. Apesar de sua efetividade, muitas reações adversas têm sido relatadas que incluem desordens gastrointestinais, leucopenia, desenvolvimento de metemoglobinemia com cianose e anemia hemolítica em

1. Gerência de Malária, Fundação de Medicina Tropical do Amazonas, Manaus, AM. 2. Universidade do Estado do Amazonas, Manaus, AM. 3. Centro Universitário Nilton Lins, Manaus, AM.

Apoio: Fundação de Medicina Tropical do Amazonas (FMTAM) e Fundação de Amparo à Pesquisa do Estado do Amazonas (FAPEAM).

Endereço para correspondência: Dr ${ }^{a}$ Marli Stela Santana Maciel. Gerência de Malária/FMTAM. Av. Pedro Teixeira 25, Bairro D. Pedro I, 69040-000 Manaus, AM.

Tel: 5592 2127-1243

e-mail:marli.maciel@uol.com.br

Recebido para publicação em: 24/04/2007

Aceito em: 20/08/2007 
indivíduos com deficiência hereditária da glicose-6-fosfato desidrogenase (G6PD). Portanto, é contra-indicada para gestantes e indivíduos deficientes da G6PD ${ }^{31}$.

Metemoglobinemia refere-se à oxidação do ferro da hemoglobina em estado ferroso $\left(\mathrm{Fe}^{++}\right)$ao estado férrico $\left(\mathrm{Fe}^{+++}\right)$, impedindo desta forma a hematose $\mathrm{e}^{22}$. Os eritrócitos contêm uma taxa normal de metemoglobina (1-2\%), mantida por mecanismos redutores fisiológicos, resultantes da oxidação espontânea. No entanto, a presença de metemoglobina em quantidades elevadas torna-se incompatível com a vida ${ }^{34}$. Há um grande número de agentes capazes de produzir a metemoglobina, como as aminoquinolinas, nitritos, cloratos, fenacetina, acetanilida, sulfanilamida, nitrobenzeno, anilina ${ }^{10}$, assim como compostos endógenos, nitratos, nitritos e óxido nítrico ${ }^{21}{ }^{30}$. Outros fatores podem ser associados à metemoglobinemia: acidose metabólica, principalmente em infantes ${ }^{16} 27$ 36; deficiência enzimática da cytocromo- $b 5$ redutase, citocromo $b-5$, NADPH-MHb redutase ${ }^{1220}$, e a hemoglobina $M$ ainda pode apresentar um grupo de moléculas de hemoglobina anormais ${ }^{20}$.

A toxicidade da primaquina, em relação ao seu efeito hemolítico e promoção da condição de metemoglobinemia, foi primeiramente estudada em caucasóides e negros em $1952^{817}$. Fletcher e cols ${ }^{13}$ relataram elevação dos níveis de metemoglobina em 30 indivíduos caucasóides após um regime diário de 15mg de primaquina, durante 14 dias, numa média de $6 \%$, que também foi observada em 151 indivíduos que receberam 30mg diárias num período de 20-52 semanas 3 . 0 propósito deste estudo foi verificar a associação entre metemoglobinemia e deficiência da G6PD durante 0 tratamento da infecção malárica com primaquina.

\section{MATERIAL E MÉTODOS}

Pacientes. 0 estudo foi conduzido na Fundação de Medicina Tropical do Amazonas (FMTAM), Manaus, Amazonas - Brasil, onde foram selecionados pacientes da demanda espontânea de atendimento da Gerência de Malária, com diagnóstico para malária vivax ou mista $\mathrm{V}+\mathrm{F}$ (vivax + falciparum) e da unidade hospitalar "Dr. Nelson Antunes", após concordarem com o termo de consentimento livre e esclarecido. Dois grupos de indivíduos foram recrutados: Grupo 1: 74 indivíduos com diagnóstico clínico de metemoglobinemia, em tratamento com primaquina, dentre os quais 73 com malária vivax e 1 com malária mista; Grupo 2: 161 indivíduos sem diagnóstico clínico de metemoglobinemia, em tratamento com primaquina (D10), dentre os quais $158 \mathrm{com}$ malária vivax e 3 com malária mista. 0 critério de diagnóstico clínico de metemoglobinemia seguiu os sinais e sintomas: cianose, cefaléia, tontura, dispnéia, torpor, depressão respiratória e sangue cor de chocolate $e^{2834}$.

Obtenção de amostras e métodos de laboratório. Foram coletadas amostras de sangue por punção venosa de cada indivíduo: 4,5mL em tubo contendo EDTA (ácido etilenodiaminotetrcético) para dosagem da hemoglobina, contagem de reticulócitos, com procedimentos realizados usando-se um contador Coulter (modelo STKS) e dosagem da metemoglobina pela técnica descrita por Evelyn e Malloy ${ }^{12}$, porém modificada por Naoum ${ }^{26} ; 4 \mathrm{~mL}$ em tubo com gel para dosagem da bilirrubina indireta, utilizando-se aparelho DADE Behring (modelo Dimension AR) e $6 \mathrm{~mL}$ em tubo com ACD (ácido citrato dextrose) para a dosagem qualitativa da G6PD. A atividade da G6PD foi avaliada através do teste de redução da metemoglobina, descrita por Brewer e cols5.

Análise estatística. Utilizou-se $o$ teste de Spearman ${ }^{29}$ para verificar a correlação entre os níveis de metemoglobina e de hemoglobina. Houve busca de associações estatísticas entre a metemoglobinemia e as demais variáveis, usando os testes: qui-quadrado, exato de Fisher e Kruskal-Wallis. Também foram utilizadas medidas de força da associação - risco relativo (RR) e odds ratio (OR), com utilização da tabela $2 \mathrm{X} 2$ simples - entre metemoglobinemia/não - metemoglobinemia e deficiência da G6PD/não - deficiência. As análises estatísticas foram realizadas em nível de significância de 5\% (p< $<0,05)$, intervalo de confiança de $95 \%$ e processadas com auxílio dos softwares Epi-Info versão $6.04 \mathrm{~d}^{7}$ e MINITAB versão $13^{24}$.

\section{RESULTADOS}

Foram analisadas amostras de 235 indivíduos, compondo os dois grupos de estudo (Tabela 1). Os níveis de metemoglobina estiveram elevados em todos os indivíduos do Grupo 1 , alcançando média de $11,1 \%$, tendo todos os indivíduos apresentado cianose. Após comparação da média do nível de metemoglobina sanguínea do Grupo 1, foi significativamente mais elevada que no Grupo 2 ( $<<0,05$ ). Em $20 \%$ dos indivíduos com metemoglobinemia, apresentando menos que $8 \mathrm{~g} / \mathrm{dL}$, a metemoglobina apresentou-se em níveis acima de $10 \%$, havendo moderada correlação positiva na combinação de indivíduos com metemoglobinemia e suas respectivas taxas de hemoglobina $(\mathrm{r}=0,54)$. 0 número de reticulócitos esteve aumentado em $92 \%$ dos 74 indivíduos do Grupo $1 \mathrm{e}$ a bilirrubina indireta aumentada de forma significante nos indivíduos que eram deficientes da G6PD, havendo significância na relação destas variáveis com a metemoglobinemia $(\mathrm{p}<0,05)$. A diminuição dos valores do hematócrito e hemoglobina, quando relacionada à metemoglobinemia também apresentou significância estatística $(\mathrm{p}<0,05)$.

Tabela 1 - Características dos grupos de estudo.

\begin{tabular}{lcc}
\hline & Grupo 1 (74)* & Grupo 2 (161)* \\
\hline Idade (anos) ${ }^{* * *}$ & $23(3-67)$ & $39,3(4-77)$ \\
Sexo (\%) & masc $(81,1) / \mathrm{fem}(18,9)$ & masc $(68,9) / \mathrm{fem}(31,1)$ \\
Espécie de malária (\%) & $\mathrm{V}(98,6) / \mathrm{M}(1,4)$ & $\mathrm{V}(98,1) / \mathrm{M}(1,9)$ \\
Hemoglobina (g/dL)** & $9(4,4-15,2)$ & $11,7(6,7-15,5)$ \\
Reticulócitos (\%)*** & $5,3(1,5-15)$ & $2,4(0,4-13,8)$ \\
Bilirrubina Indireta (mg/dL)*** & $1,6(0,08-9,5)$ & $0,6(0,09-6,6)$ \\
Metemoglobina (\%)** & $11,1(2,3-38)$ & $1,8(0,4-5)$ \\
G6PD deficiente (\%) & $51,3(38)$ & $8,7(14)$ \\
\hline
\end{tabular}

*número de indivíduos incluídos em cada grupo

** média (escala entre parênteses)

masc: masculino, fem: feminino

V: malária vivax

M: malária mista (vivax + falciparum) 
Quanto à deficiência da G6PD, nos Grupos 1 e 2 (Figura 1), houve 38 e 14 indivíduos enzimopênicos, respectivamente, demonstrando através de tais dados, significância estatística na associação com a metemoglobinemia somente nos indivíduos do Grupo $1(\mathrm{p}<0,05)$. Os indivíduos com deficiência da G6PD apresentaram um odds ratio de 11,8 para metemoglobinemia, quando comparados com os que não eram deficientes. Os indivíduos com deficiência da G6PD apresentaram risco para metemoglobinemia 3,71 vezes maior do que os não deficientes. No momento da inclusão e análise dos indivíduos, não foram relatadas quaisquer evidências de associação da clínica de metemoglobinemia à infecção pelo Plasmodium.

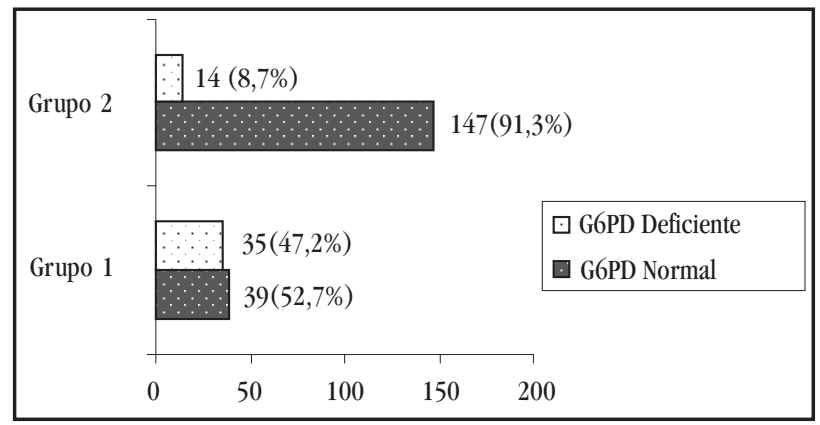

Figura 1 - Freqüência de indivíduos que apresentaram deficiência da glicose-6-fosfato desidrogenase.

\section{DISCUSSÃO}

A metemoglobinemia aguda, embora rara, pode tornar-se uma complicação potencialmente fatal, se atingir níveis acima de $70 \%{ }^{34}$. Todos os agentes potencialmente causadores devem ser suspensos imediatamente. No entanto, freqüientemente, o agente metemoglobinizante pode não ser prontamente identificado.

Estudos têm demonstrado a toxicidade da primaquina, que dentre uma variedade de efeitos adversos encontram-se a formação de metemoglobina e anemia hemolítica em indivíduos suscetíveis. Muitos esforços em substituir a primaquina por antimaláricos análogos em seu efeito gametocida e hipnozoiticida, têm sido desprendidos por mais de quatro décadas. Anstey e cols ${ }^{2}$ demonstraram que a ingestão de cloroquina não é capaz de promover a elevação dos níveis de metemoglobina, podendo, no entanto, haver aumento significativo durante a infecção por Plasmodium, no caso da malária em seus padrões de gravidade ${ }^{32}$. Radicais oxidantes gerados em hemácias parasitadas também podem causar a elevação dos níveis de metemoglobina. Um estudo in vitro demonstrou haver elevação dos níveis de metemoglobina em hemácias parasitadas pelo Plasmodium falciparum $^{15}$. 0 fato dos sujeitos desta pesquisa serem indivíduos com malária não interferiu nos resultados relacionados à metemoglobina circulante, pois nenhum destes apresentou malária grave.

Neste trabalho, a verificação da relação da metemoglobinemia e deficiência da G6PD mostrou haver uma possível associação de indivíduos enzimopênicos serem mais susceptíveis ao desenvolvimento de metemoglobinemia $(\mathrm{p}<0,05)$. Em relação à verificação de risco para a metemoglobinemia, encontrou-se uma forte associação entre essa condição clínica e a deficiência da G6PD, como a maioria da literatura aponta ${ }^{6935}$. Por outro lado, cabe ressaltar que apesar de apresentarem clínica de metemoglobinemia, nem todos os indivíduos do Grupo 1 tiveram seus níveis de metemoglobina acima da concentração mínima esperada, relatada na literatura científica, para início das manifestações clínicas, ou seja, valores acima de $15 \%{ }^{34}$. Observou-se que na presença de nível de metemoglobina três vezes acima do normalmente circulante (2,3\% de metemoglobina num indivíduo com taxa normal de $0,5 \%)$ há possibilidade de ocorrer a uma condição de metemoglobinemia clínica. Há, no entanto, necessidade de investigar a possibilidade de fatores não inerentes à toxicidade da primaquina estarem envolvidos, como fatores fisiológicos e genéticos.

A triagem da deficiência da G6PD realizada pelo método de Brewer ${ }^{5}$ pode ter limitado a frequiência de indivíduos enzimopênicos, principalmente nos indivíduos do Grupo 1, visto que a aplicação do mesmo em condição de reticulocitose pode interferir no diagnóstico da deficiência da G6PD, pois os reticulócitos são ricos em G6PD ${ }^{25}$, podendo apresentar um resultado de falsa atividade da G6PD. 0 mesmo resultado pode ter ocorrido nos indivíduos transfundidos com hemácias de atividade normal, após crise hemolítica, que foi o caso de alguns indivíduos do Grupo 1.

Em relação à hiperbilirrubinemia indireta encontrada nos indivíduos do presente estudo, representa um achado importante, quando associado à metemoglobinemia. Resultados de uma investigação realizada em neonatos demonstraram que a intensa formação de óxido nítrico, liberação de ferritina - ferrosa $\left(\mathrm{Fe}^{+2}\right)$ e acúmulo de metemoglobina no sangue intensificam o estresse oxidativo, levando a uma fragilidade da membrana dos eritrócitos, induzindo hemólise e tendo como principal consequiência, uma condição clínica de hiperbilirrubinemia ${ }^{1}$.

Dados importantes da literatura têm demonstrado que a deficiência da G6PD também deve ser considerada quando relacionada a outros fatores hereditários como a mutação no gene codificador uridinodifosfoglucuronato glucuronosiltransferase 1 (UDPGT1) afetada na síndrome de Gilbert, diante de um processo de estresse oxidativo, quando há maior risco de ocorrência de hiperbilirrubinemia grave gr1 $^{118}$.

Quanto aos indivíduos do Grupo 2, não ocorreram alterações significativas, nem mesmo naqueles que eram deficientes enzimáticos, quando tratados com primaquina.

\section{AGRADECIMENTOS}

Os autores agradecem a todos que cooperaram na execução desta produção científica, à Fundação de Medicina Tropical do Amazonas (FMTAM), através da Gerência de Malária e Programa de Apoio à Iniciação Científica (PAIC), que atuou como entidade executora e financiadora, e à Fundação de Amparo à Pesquisa do Estado do Amazonas (FAPEAM) pelo apoio financeiro.

\section{REFERÊNCIAS}

1. Abzhandadze TIu, Kvezereli-Kopadze AN, Dzhaparidze ESh, Tkhilava NG, Sanikidze TV. The role of oxidative metabolism in pathogenesis of hyperbilirubinemia in infants. Georgian Medical News 136:77-80, 2006. 
2. Anstey NM, Hassanali MY, Mlalasi J, Manyenga D, Mwaikambo ED. Elevated levels of methaemoglobin in Tanzania children with severe and uncomplicated malaria. Transactions of the Royal Society of Tropical Medicine and Hygiene 90: 147-151, 1996.

3. Baird JH, Fryauff DJ, Hoffman SL. Primaquine for prevention of malaria in travelers. Clinical Infection Disease, 37:1659-1667, 2003.

4. Beutler E, Gelbart T, Miller W. Severe jaundice in a patient with a previously undescribed glucose-6-phosphate dehydrogenase (G6PD) mutation and Gilbert syndrome. Blood Cells Molecules and Diseases 28:104-107, 2002.

5. Brewer GJ, Tarlov AR, Alving AS. The methemoglobin reduction test for primaquine-type sensitivity of erythrocytes. A simplified procedure for detecting a specific hypersusceptibility to drug hemolysis. Journal of American Medicine Association 180: 386-388, 1962

6. Browning LA, Kruse JA. Hemolysis and methemoglobinemia secondary to rasburicase administration. The Annals of Pharmacotherapy 39:1932-1935, 2005.

7. Centers for Disease Control \& Prevention. Epi-Info version 6.04d. CDC, Atlanta, 2001.

8. Clayman CB, Arnold J, Hockwald RS, Yount Jr EH, Edgcomb JH, Alving AS. Toxicity of primaquina in Caucasians. Journal of the American Medical Informatics Association 149:1563-1568, 1952

9. Coleman MD, Coleman NA. Drug-induced methaemoglobinaemia. Treatment issues. Drug Safety: an International Journal of Medical Toxicology and Drug Experience 14:394-405, 1996.

10. Coleman MD, Coleman NA. Drug-induced methaemoglobinemia: etiology, pharmacology, and clinical management. Annals of Emergency Medicine 34:646656, 1999.

11. Edwards CQ. Anemia and the liver. Hepatobiliary manifestations of anemia. Clinics in Liver Disease 6:891-907, 2002

12. Evelyn KA, Malloy HT. Microdetermination of oxyhemoglobin, methemoglobin and sulfhemoglobin in a single sample of blood. Journal of Biological Chemistry 126:655-657, 1938

13. Fletcher KA, Evans DA, Gilles HM, Greaves J, Bunnag D, Harinasuta T. Studies on the pharmacokinetics of primaquine. Bull World Health Organization 59:407-412, 1981.

14. Fundação Nacional de Saúde. Manual de Terapêutica da Malária. Vigilância Epidemiológica, Brasília 12: 30-33, 2001.

15. Golenser J, Kamyl M, Tsafack A, Marva E, Cohen A, Kitrossky N, Chevion M. Correlation between destruction of malarial parasites by polymorphonuclear leucocytes and oxidative stress. Free Radical Research Communication 17:249262,1992

16. Hjelt K, Lund JT, Scherling B, Bendixen S, Lundstrom K, Stovring S, Voldsgaard P, Linnet K. Methemoglobinemia among neonates in a neonatal intensive care unit. Acta Pediatrica 84:365-370, 1995.

17. Hockwald RS, Arnold J, Clayman CB, Alving MD. Toxicity of primaquine to negroes. Journal of American Medicine Association 149:1568-1570, 1952.

18. Huang CS, Huang MJ, Lin MS, Yang SS, Teng HC, Tang KS. Genetic factors related to unconjugated hyperbilirrubinemia amongst aduslts. Pharmacogenetics and Genomics 15:43-50, 2005
19. Jaffe ER. Enzymopenic hereditary methemoglobinemia: a clinical/biochemica classification. Blood Cells 12:81-90, 1986.

20. Jaffe ER, Hultquist DE. Cytochrome b5 Redutase Deficiency and Enzymopenic Hereditary Methemoglobinemia. In: Scriver CR, Beaudet AB, Sly WS, Valle D (eds) The Metabolic Basis of Inherited Disease, McGraw Hill, New York, p.22672280, 1989.

21. Lukens JN. Metemoglobinemia e Outros Distúrbios Acompanhados por Cianose. In: Lee GR, Bithell TC, Foerster J, Athens JW, Lukens JN (eds) Wintrobe Hematologia Clínica, 1ª edição, Manole, São Paulo, p.1386-1395, 1998.

22. Mansouri A, Lurie AA. Concise review. Methemoglobinemia. American Journa of Hematology 42:7-12, 1993

23. Ministério da Saúde. Situação Epidemiológica da Malária no Brasil. Disponível na URL: portal.saude.gov.br/portal/arquivos/pdf/be_malaria_01_2005.pdf, Acessado em 20 de fevereiro de 2007. Brasília, 2005.

24. Meet Minitab. MINITAB, INC. Release 13 for Windows. State College, PA, 2000 .

25. Miyazono Y, Hirono A, Miyamoto Y, Miwa S. Erythrocyte enzyme activities in cord blood of extremely low-birth-weight infants. American Journal of Hematology 62:88-92, 1999.

26. Naoum PC. Eletroforese, Técnicas e Interpretação. $2^{\text {a }}$ edição, Editora Santos, São Paulo, p.89-91, 1999

27. Pollack ES, Pollack Jr V Incidence of subclinical methemoglobinemia. in infants with diarrhea. Annals Emergency of Medicine 24:652-656, 1994.

28. Rodriguez LF, Smolik LM, Zbehlik AJ. Benzocaine-induced methemoglobinemia: report of a severe reaction and review of the literature. Annals of Pharmacotherapy 28:643-649, 1994

29. Sachs L. The comparation of independent data. In: Sachs L (ed) Applied Statistics A Handbook of Techniques. $2^{\text {nd }}$ edition, Verlag Spring, New York, p. 299-303, 1984

30. Wennmalm A, Bentin G, Edlund A, Jungersten L, Kieler-Jensen N, Lundin S, Westfelt UN, Petersson A, Waagstein F. Metabolism and excretion of nitric oxide in humans. Circulation Research 73:1121-1127, 1993.

31. World Heath Organization. Practical chemotherapy of malaria. Thechnical report series 805 . Geneva, p. 34-35, 1990.

32. World Heath Organization. Management of Severe Malaria. A practical handbook $2^{\text {nd }}$ edition. Disponível na URL: http://who.int/malaria/docs/ hbsm_toc.htm. Acessado em 20 de setembro de 2005. Geneva, 2000.

33. World Health Organization. Roll Back Malaria. World Malaria Report. Disponível na URL: http://www.rbm.who.int/wmr2005/html. Acesso em 20 fevereiro de 2007, 2005

34. Wright R, Lewander W, Woolf A. Methemoglobinemia: etiology, pharmacology, and clinical management. Annals of Emergency Medicine 34:646-655, 1999.

35. Yang CC, Wu ML, Deng JF. Prolonged hemolysis and methemoglobinemia following organic copper fungicide ingestion. Veterinary and Human Toxicology 46:321323,2004

36. Yano SS, Danish EH, Hsia YE. Transient methemoglobinemia with acidosis in infants. Journal Pediatric 100:415-418, 1982 